\title{
Response of a mass-spring system subject to Coulomb damping and harmonic base excitation
}

\author{
Daniel J. Riddoch *, Alice Cicirello, David A. Hills \\ Department of Engineering Science, University of Oxford, \\ Parks Road, Oxford, OX1 3PJ, United Kingdom
}

\begin{abstract}
The periodic response of a mass-spring system is studied and the behaviour described, including the creation of finite dwell periods. A parameter space investigation is conducted and a branch of behaviour with the space is explained. A new solution method is proposed for direct calculation of the steady-state response without calculating a transient phase. A new approach to the display of the steady-state response is given. Finally, the system behaviour near its resonance is explored and new asymptotic solutions are proposed for the response amplitude near resonance. The accuracy of these solutions is then determined by comparison to the true solution.
\end{abstract}

Keywords - Stick-slip motion, Steady-state, Asymptotic solution, Friction, Coulomb damping

\section{Introduction}

Unlubricated (dry) joints abound in both mechanical assemblies and in, for example, structural steelwork. The components fastened together are intended to be truly stationary, with no relative motion, but often the applied loads present will excite shear tractions along the interface, and frictional forces are insufficient to prevent at least some slip. The literature is replete with analysis of so-called 'partial slip' contacts subject to harmonic loading[1, 2, 3, 4], and deals with quasi-static analysis of their response, usually in order to probe the possibility of fretting damage and the nucleation of cracks. But it is important to realise that oscillatory frictional contacts also provide damping, and remove energy, so limiting the amplitude of vibration experienced. Equally, buildings are subject to seismic excitation, and the connections between structural elements again provide damping.

In some problems, for example in the cases of fanblades in gas turbines, although the contact may be 'partial slip' in nature, it not infrequently slides fully, and the question arises of how the system will respond. So far, there have been very few attempts to analyse the behaviour of problems with frictional damping, and here we look at a very simple, idealised problem so as to gain insight into the likely response of more complicated problems. No attempt is made to introduce partial slip, and the inertial mass is assumed to be perfectly rigid.

We will consider, here, a simple system with a rigid mass, $m$, resting on a frictional surface and where the limiting frictional force is of magnitude $F_{f}$, which will often be the product $\mu m g$ where $\mu$ is the coefficient of friction and $g$ the acceleration due to gravity, but we maintain this notation, for the time being, as in some cases the frictional force may be externally imposed. We distinguish between three similar configurations, Figure 1. In Figure 1(a) we show the simplest arrangement where an external force $F(t)$, where $t$ is time, is applied directly to the mass. Related problems in which the excitation

\footnotetext{
*Corresponding author: Tel.: +44 1865273811 ;

E-mail address: daniel.riddoch@eng.ox.ac.uk (D. Riddoch).
} 
is by imposed displacement, $y(t)$, at the end of the spring are shown in Figure 1(b,c). In the first of these the surface on which the mass is resting is also referred to the position $y(t)$, and in the second the frictional force is referred to stationary ground. In this paper the problem shown in Figure 1(c) is studied, and harmonic excitation is used throughout.

The seminal paper in this area is that of Den Hartog [5] from 1930. The key results of his study are establishing the boundary, in the steady-state, between continuous motion and one where stick-slip motion occurs, and the magnitude of the response in the former case. More recently, a detailed numerical investigation was conducted by Hong and Liu [6] who considered and categorised the multi-stop behaviour of the system. Papangelo and Ciavarella [7] concentrated on driving frequencies frequency much smaller than the natural frequency with a different number of stops. All three of these authors analysed the imposed force problem, as shown in Figure 1(a). Others have distinguished between the dynamic and static coefficients of friction [8], and lastly, in [9], a further configuration was looked at where the mass rests on a continuously moving belt, connected by a spring to a fixed wall. The case of a seismic system, as shown in figure 1b, has been investigated by [10] and an extension to Den Hartog's work is presented.

So what we aim to do here is to classify the response in the stick/slip region, to determine how many times during a steady state cycle the mass stops, to determine the transmissibility of displacement. This is all done for the simplest model, discussed further in section 2. We also aim to improve or simplify where possible, such as around resonance, the work of Den Hartog. We do this by applying symmetric conditions to find the steady state behaviour, and by applying asymptotic methods to the existing solutions.

The paper is laid out as follows; in section 2.1 the results of the Den Hartog paper are discussed further and a new graphical procedure is introduced. In section 3, the response of the system during stick slip motion is discussed and explained, this is extended to multiple stop cases in section 3.1. A feature of the parameter space response is identified and discussed in section 4 for the first time and the behaviour of the system near resonance is examined thoroughly in section 6 . Some comments are made about energy dissipation and work done in section 7 .

\section{Formulation}

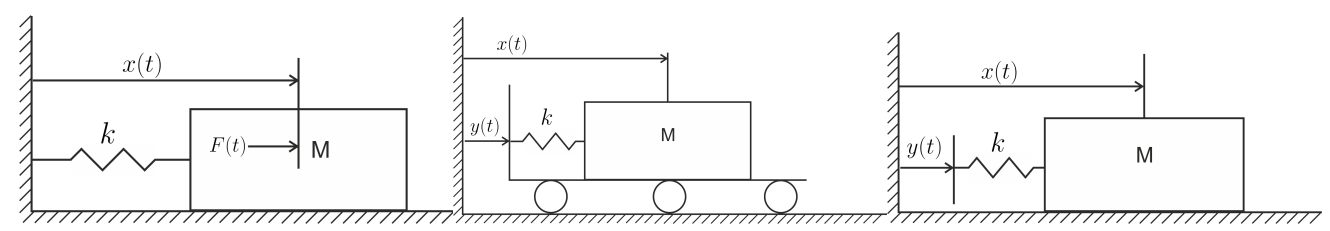

(a) Force excited

(b) Seismic excitation

(c) Base excitation

Figure 1: Coulomb friction damper subject to a base excitation

Refer to Figure $1(\mathrm{c})$. The force in the spring, $F_{s}$, is given by

$$
F_{s}=k(y-x),
$$

and, if both the magnitude of this force is less than the limiting frictional force $\left(\left|F_{s}\right|<F_{f}\right)$ and the mass is stationary $(\dot{x}=0)$ it will continue to be at rest. It will be in equilibrium and the spring force will be precisely opposed by the frictional force.

Figure 2 displays behaviour of the system in dimensionless displacement space and figure $2(\mathrm{a})$ shows the quasi static response, figures $2(\mathrm{~b}-\mathrm{d})$ show the dynamic response of the system. If the mass is positioned anywhere within the red lines it will remain stationary. If the amplitude of forcing is such that the limiting frictional force is reached, and it varies harmonically in time but at a very low frequency so that inertia forces are very small, the mass will track out a loop of the form shown in blue. 


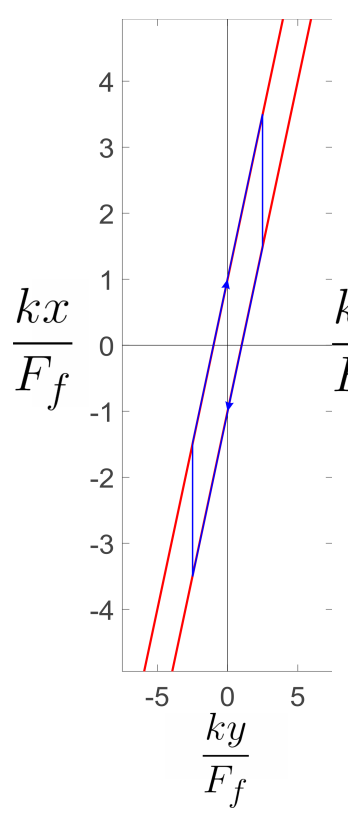

(a) Quasi-static

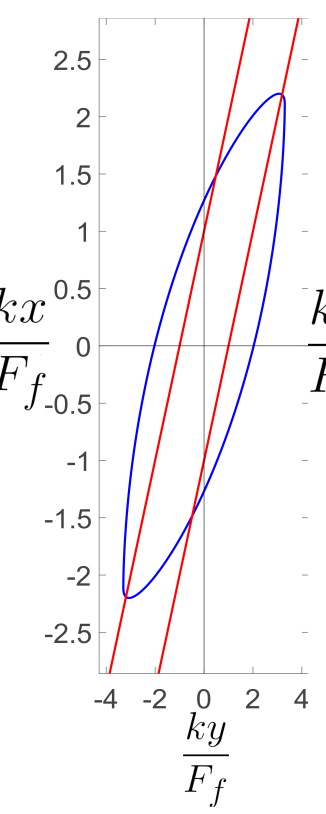

(b) Dynamic, $\frac{k Y}{F_{f}}=1.1$

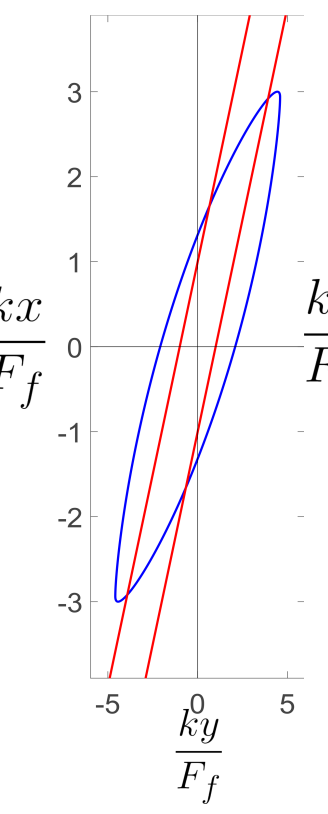

(c) Dynamic, $\frac{k Y}{F_{f}}=1.5$

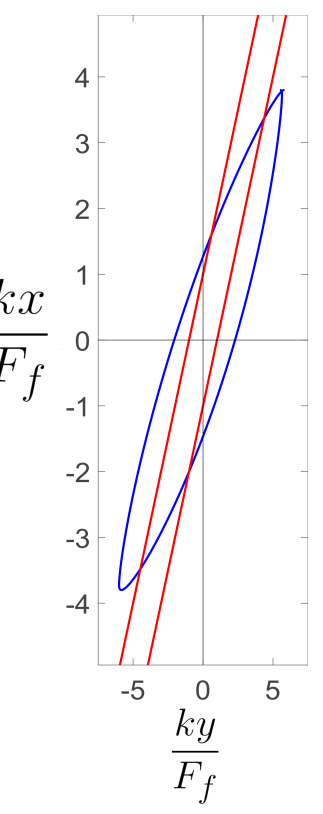

(d) Dynamic, $\frac{k Y}{F_{f}}=1.9$

Figure 2: Dimensionless plots of base input against response showing quasi-static and dynamic response

If either the spring force exceeds the limiting frictional force $\left(\left|F_{s}\right|>F_{f}\right)$ or the mass is in motion $(|\dot{x}|>0)$, or, indeed, if both of these inequalities hold, the mass has a net force on it causing it to accelerate, and application of Newton's second Law of Motion gives

$$
F_{s}-F_{f} \operatorname{sgn}(\dot{x})=m \ddot{x}
$$

or

$$
\ddot{x}+\omega_{n}^{2}(x(t)-y(t))+\frac{F_{f}}{m} \operatorname{sign}(\dot{x})=0
$$

where $\operatorname{sgn}($.$) is the signum function, and we can now see that the physical problem is characterised by$ two quantities, the natural frequency of the problem, $\omega_{n}^{2}=\frac{k}{m}$, and the ratio of the frictional force to the inertial mass, $F_{f} / m \equiv p$, and which would be given by $\mu g$ when the frictional force originates in the weight of the mass.

We now restrict our attention to harmonic forcing, and choose $y(t)=Y \cos \left(\omega_{b} t+\phi\right)$ where the "phase shift', $\phi$, is included here to simplify the ensuing algebra. When refering to a single cycle we shall measure the time from the maximum of the base displacement to the subsequent maximum, thus the phase shift will be displayed in the response of the mass. The general solution to differential equation (1) is given by

$$
\frac{x(t)}{Y}= \begin{cases}\frac{A}{Y} \cos \left(\omega_{n} t\right)+\frac{B}{Y} \sin \left(\omega_{n} t\right)+\frac{p}{Y \omega_{n}^{2}}+\frac{\cos \left(\omega_{b} t+\phi\right)}{1-r^{2}} & \dot{x}>0 \\ \frac{A}{Y} \cos \left(\omega_{n} t\right)+\frac{B}{Y} \sin \left(\omega_{n} t\right)-\frac{p}{Y \omega_{n}^{2}}+\frac{\cos \left(\omega_{b} t+\phi\right)}{1-r^{2}} & \dot{x}<0\end{cases}
$$

where $r$ is the dimensionless forcing frequency $r=\frac{\omega_{b}}{\omega_{n}}$, and $\frac{Y \omega_{n}^{2}}{p}$ represents the dimensionless magnitude of forcing. Also at this point, we define $T=\frac{2 \pi}{\omega_{b}}$, the period of the steady-state motion.

At this point, it should be noted that equation 2 is the solution to equation 1 , which is an equation of motion, and hence it is valid only when the mass is in motion. In section 3 we describe responses with finite periods of arrest, when the mass is stationary and we cannot apply these equations throughout. 
Hence, when the solution incorporates periods of dwell, their beginning and end points, together with the preserved displacement of the mass, must be found.

\subsection{Existing solution}

The motion of the mass may be expected to display a transient phase, and then to settle down into a steady-state periodic motion, and it is this which is of primary interest here. The dimensionless amplitude of forcing is given by the group $\frac{Y \omega_{n}^{2}}{p}$ and we note that, if the mass is already stationary, it will remain so if this quantity is less than unity. We also state, without proof, that even if the mass is initially moving it will become stuck within a finite time when this holds true.

Although Den Hartog [5] studied a slightly different problem, figure 1a, some of the results he found are relevant to the problem at hand. He used boundary conditions, described in appendix A.1 to show that, when the amplitude of forcing is large enough the mass will be in continuous motion, the maximum displacement of which in the steady state, $X$ from here on referred to as the amplitude, is given by

$$
\left(\frac{X}{Y}\right)^{2}=\left(\frac{1}{1-r^{2}}\right)^{2}-\left(\frac{p \sin \left(\frac{\pi}{r}\right)}{Y r \omega_{n}^{2}\left(1+\cos \left(\frac{\pi}{r}\right)\right)}\right)^{2} .
$$

The resulting motion is very complicated. It is periodic, and the corresponding angular frequency is $\omega_{b}$, but within an individual cycle there is content at the natural angular frequency, $\omega_{n}$, and it is not possible to provide a description of the waveform in closed form.

If the amplitude of the forcing function is gradually reduced, there comes a point at which the mass halts at some point during the cycle. The boundary between continuous and interrupted motion is found by enforcing the condition that the sign of the velocity must remain the same at all instants within any half cycle (and must change sign between consecutive half cycles) and enforcing the symmetry and continuity of displacement by use of periodic conditions. The requirement that the velocity not change sign in any half cycle becomes

$$
\left(\frac{k Y}{F_{f}}\right)^{2} \geq\left(1-r^{2}\right)^{2}\left({\frac{S}{r^{2}}}^{2}+\frac{\sin ^{2}\left(\frac{\pi}{r}\right)}{r^{2}\left(1+\cos \left(\frac{\pi}{r}\right)\right)^{2}}\right) .
$$

where $S$ is the maximum value in the time interval $0<t<\pi / \omega_{b}$ of

$$
\frac{r \sin \left(\frac{\pi}{r}\right)+\frac{\sin \left(\frac{\pi}{r}\right)}{1+\cos \left(\frac{\pi}{r}\right)}\left(\cos \omega_{b} t-\cos \omega_{n} t\right)}{\sin \omega_{b} t} .
$$

These results allow us to introduce figure 3, which we will refer back to several times throughout this paper. The results shown in figure 3 are found only for the specific value of $\mu$ of 0.3875 . Figure $3(\mathrm{a})$ shows the number of stops per cycle of the steady state, and we have already defined the boundaries between (i) permanent stick and motion, and (ii) the boundary between continuous motion and motion with at least one period of dwell. Figure 3(b) shows the amplitude of response in the steady state, normalised by the magnitude of excitation, $\frac{X}{Y}$. Its value has already been stated (equation 3 ).

The boundaries between fully stuck and interrupted motion solutions, and between interrupted and continuous motion which are shown here can be calculated directly for any value of the coefficient of friction. The boundary between interrupted and continuous motion is described by equation 4 , and the boundary between interrupted motion and fully stuck solutions is given by $k Y=p m$.

Figure 3 is found using a meshgrid of parameter values, with the values at each point being calculated using the method described in section 3.1 and Appendix A. The 'jagged' appearance is due to the resolution of this meshgrid and interpolation performed between the points of the grid. Note that the six stops conditions only cover a small region on subfigure (a), coordinates of such a case are $r=0.1$, $\frac{k Y}{F_{f}}=0.6$. 

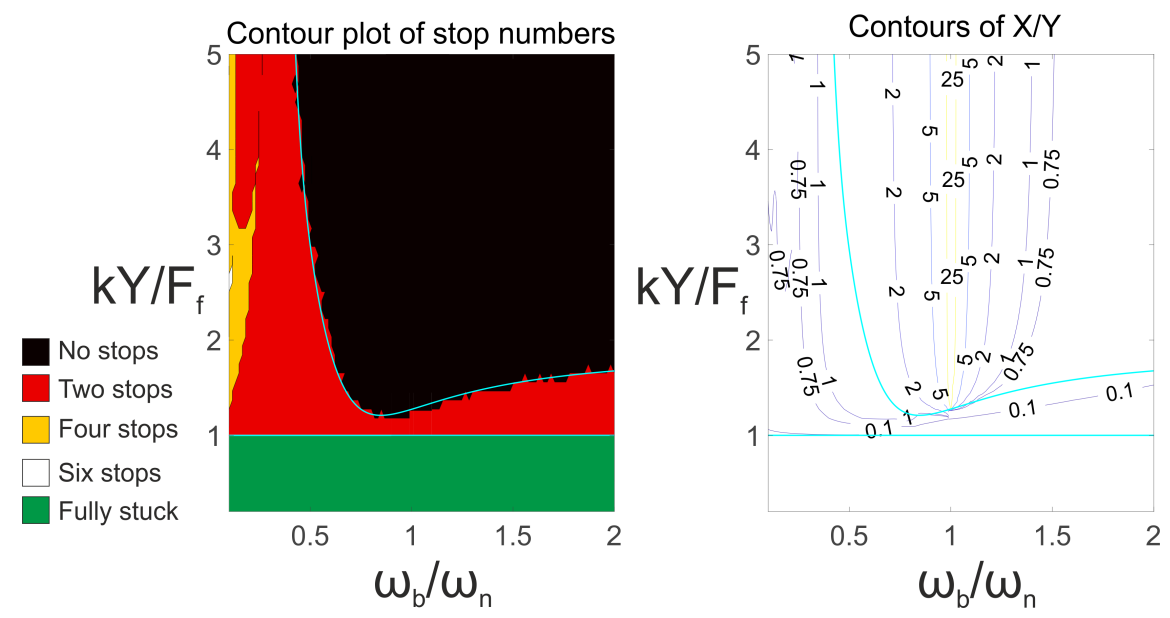

Figure 3: The parameter space response of the system at the value $\mu=0.3875$. Subfigure (a) shows the number of finite dwell periods per steady state cycle, subfigure (b) shows the dimensionless amplitude of displacement response, $X / Y$.

\section{The Stopping Problem}

Recall that, in section 2, we state that the equation of motion(equation 1) does not apply during dwell periods. Hence, a marching in time solution is initially sought to determine the system response in cases of finite dwell periods. Using this solution a transient phase is calculated and the steady-state response is reached subsequently. In cases away from the system resonance, this is achieved quite quickly. We have reviewed the existing steady-state solution, appropriate when the mass comes to rest only instantaneously at the extremes of displacement. We now describe the solution of the problem when there are finite periods of dwell. The principles adduced will generally apply when there is interrupted motion, but the method becomes unwieldy as the number of stops increases and it is, in any case, most instructive to look at the 'two-stop' case first. This corresponds to the region coloured red in figure $3(\mathrm{a})$, which the reader may observe covers a large area and a diverse range of parameter values. Starting from the point of a case with no stops of finite duration and now gradually increasing the coefficient of friction, we observe that two finite stops develop at the extremes of the base displacement. At the beginning of these stop periods, the velocity reaches zero and the value of the force in the spring is not sufficient to cause sliding. So the mass becomes stuck, and the frictional force balances the force in the spring. At the end of these stop periods, the force in the spring reaches the limiting value of the friction as the spring is stretched or compressed further by the continuing base displacement. Note that, the reference point in time is changed here, from the maximum value of the response to the amplitude of the base displacement, this is now carried throughout the paper from here. This has been done as there is no distinct peak of the response, as this is where the dwell periods are to be found.

The development of these two periods of finite dwell is best seen in cases near to the boundary between continuous and interrupted motion, which figure 4 is an example of. The top plot shows the force in the spring in pink, with the limiting value of the friction in black. The subsequent plots show the displacement, velocity and acceleration of the mass, normalised by the amplitude of the base displacement, velocity and acceleration respectively. Note the discontinuity in acceleration at the points where the velocity changes sign (and hence the sense of the spring force reverses).

By contrast with this, we can see in figure 5 a typical two stop response. The two finite stop regions can be seen between the red vertical lines. The two finite dwell periods are where the extremes of motion were.

A set of boundary conditions defining the periodic behaviour was developed. This is described in full and algebraically in appendix A.2. This method is much faster, for individual cases, than traditional 


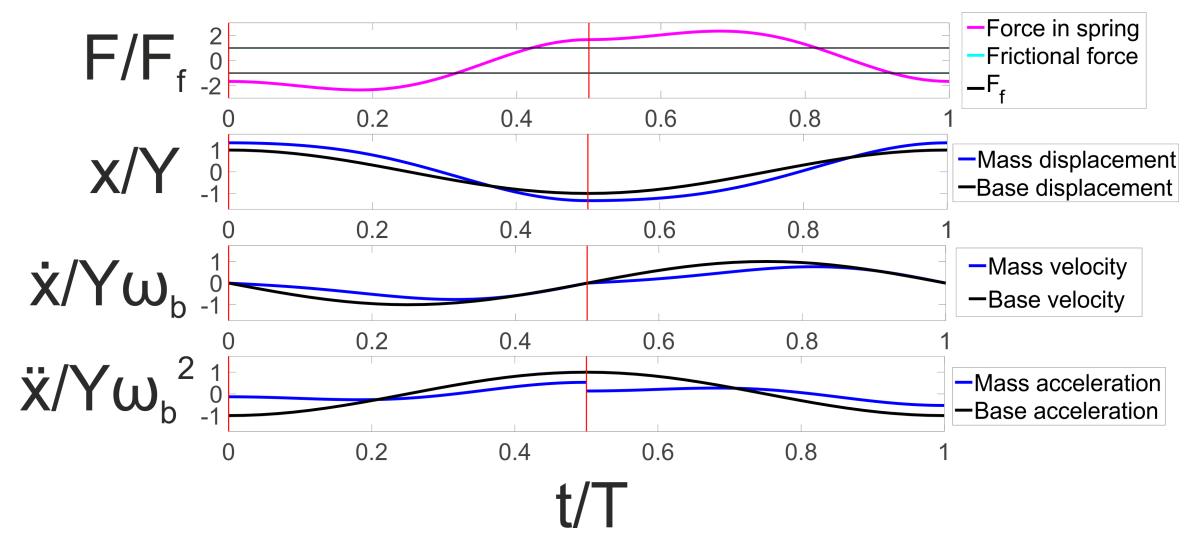

Figure 4: A typical system in continuous motion, $\frac{k Y}{F_{f}}=5, r=0.5$

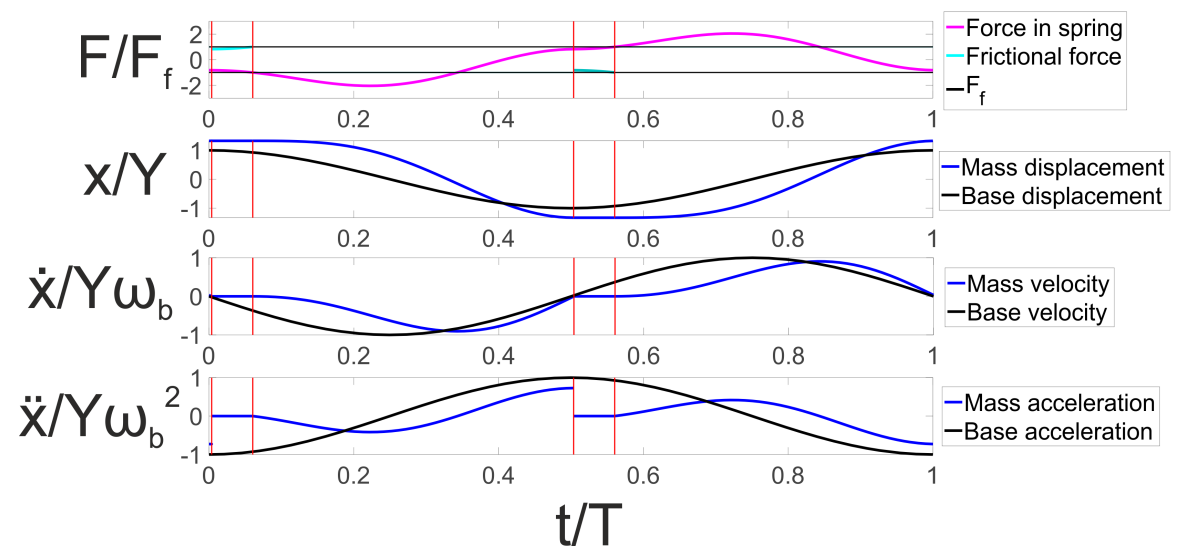

Figure 5: A typical response of a two stop system, $\frac{k Y}{F_{f}}=2.5, r=0.5$

time marching solutions. However, as the problem is underspecified by the equation of motion alone, the solvers are not reliable around the boundaries between continuous and interrupted motion, and motion with different numbers of finite dwell periods.

\subsection{The multiple stop problem}

As previously stated, the principles above can be extended to cases with multiple stops. In the 'four-stop' system there are four finite dwell periods per cycle of the base displacement in the steady state. Intermediate stops develop when the ratio between the driven and natural frequencies is small, which gives rise to multiple peaks of the force in the spring. These intermediate peaks and their corresponding troughs become larger, with either a reduction of the frequency ratio or an increase in the input amplitude. An example of one of these cases can be seen in figure 6 .

The boundary conditions and solution method can be extended to cover problems with more than two stops, as described in appendix A.3, but the system remains underspecified and the method becomes unwieldy. This presents a problem exploring regions with a very small excitation frequency compared to the natural frequency, as these cases have lots of sub-resonances, giving rises to many stops per cycle. This region was explored in more detail by Papangelo and Ciavarella [7]. 


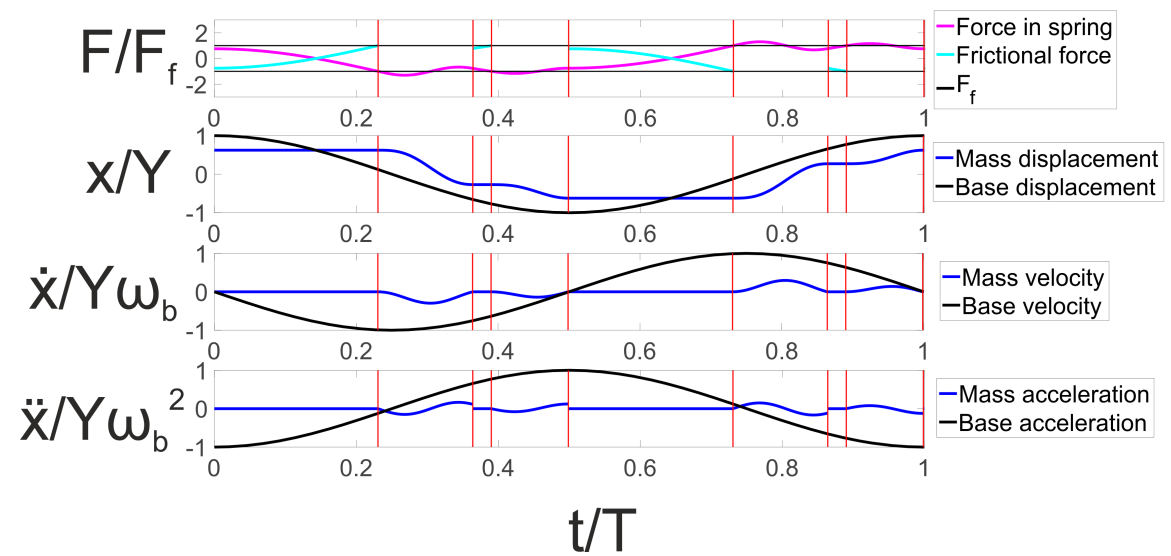

Figure 6: A typical four stop system, $\frac{k Y}{F_{f}}=1.5, r=0.1$

\section{The stop branch}

Referring back to figure 3(a), it can be seen that there is an isolated 'branch' of input parameters which lead to more finite dwell periods than other cases around them, such as around the point $r=0.25$, $\frac{k Y}{F_{f}}=4$. We shall refer to this as the stop branch. We seek to explain the reasons that this branch exists and to do this, we use the four stop branch, shown in yellow on figure 3(a), as an illustrative case. A set of example plots is shown in figure 8 , where figure 7 is a zoomed on part of figure $3(\mathrm{a})$, and figure 8 1-5 represent various cases around the stop branch.

To simplify the explanation, we separate the changes into frequency ratio and input amplitude variations, and consider them separately. Initially, consider a constant frequency ratio, and different cases of input amplitude. It can be observed that increasing the amplitude directly increases the response amplitude. As such, in figure 8(4), the amplitude of the second, local maxima in the force in the spring in not sufficient to cause sliding. This maxima becomes large enough to cause sliding in (2), hence we get a second period of sliding, whereas the minima between the two maxima is not small enough to cause stick in (3), so we do not get a period of stick.

Now consider, alternatively, the case of changing the frequency of excitation only. Increasing the driving frequency changes the ratio between the magnitude of the maxima described above. In the same way that changing the magnitude of the maxima causes the restart to occur and then the minima to become sufficiently large as to prevent slip, changing the magnitude of the second maxima has the same effect. As can be seen by comparing figure 8 (5), (2) and (1).

\section{Display of Behaviour over one Cycle}

We introduced a method of displaying the steady state behaviour with figure 2, for the quasi-static solution and cases of continuous motion. Applying this method to dynamic cases in which the steady state solution has multiple periods of finite dwell per half-cycle, this display regime shows more information. We compare the graphical procedure for cases with no finite stops, two and four finite stops per cycle in figure 9. It can be seen that the number of distinct sections in which the response is shown as a vertical corresponds to the number of finite stops per cycle of the steady state. This must be the case, as a vertical line corresponds to no change in $x$, in other words the mass is not moving, but a change in $y$. Further, the rounded corners of the case in figure $9(\mathrm{~b})$ indicate that the mass was in motion at the extreme of the base displacement, in other words, a phase shift was present. This is the case because the extreme values of $x$ and $y$ do not align; otherwise a 'corner' would be present. 


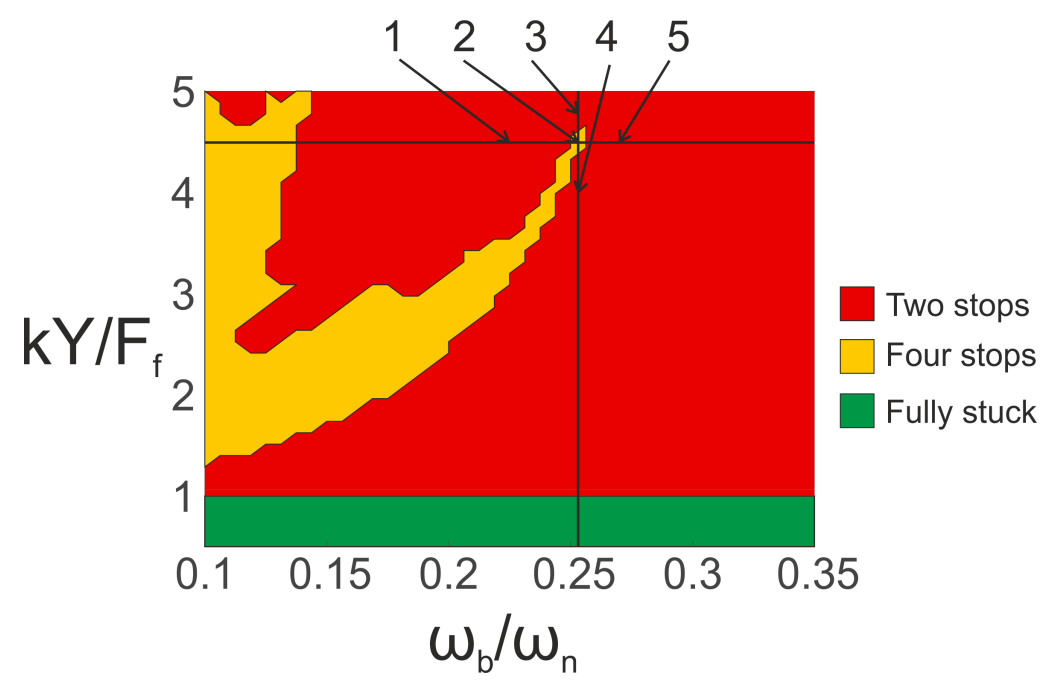

Figure 7: Closer examination of the stop branch and identification of five example cases

\section{Behaviour near resonance}

When $\frac{\omega_{b}}{\omega_{n}}=1$, the system is excited at its resonant frequency. Figure 3 shows that there are only three possible responses. Clearly, if $\frac{k Y}{F_{f}}<1$, the system is fully stuck in the steady state. If $\frac{k Y}{F_{f}}>\frac{4}{\pi}$, then the simplification of equation 4 tells us that the system is a case of continuous motion. Finally, if $1<\frac{k Y}{F_{f}}<\frac{4}{\pi}$, then the mass must display stick-slip motion, which is, in fact, two-stop motion. The amplitude of this oscillation is shown in figure 10. Its limit at the onset of continuous motion being equal to the amplitude of the base displacement. If the motion is continuous, it does not reach a limit cycle, so the amplitude grows without bound.

Using the equations set out in section 2, an asymptotic solution is sought for the steady amplitude of the system near resonance, as the ratio of the driven to the natural frequency approaches unity:

$$
X=\frac{Y \cos \left(\frac{T \arcsin \left(\frac{4 F_{f}}{k Y \pi}\right)}{2 \pi}\right)}{1-r^{2}}
$$

The accuracy of the solution can be seen by means of a set of contours, shown in figure 11, which shows the absolute error of the asymptotic solution compared the full solution presented for no stop cases by Den Hartog.

\section{Energy balance and damping}

As the magnitude of the frictional force is constant when the block is sliding, it is clear that the work done per cycle, $W$, is directly proportional to amplitude of the response and is given by $W=4 F_{f} X$, and is consequently independent of the velocity of the mass. Therefore the damping is dependent only on the amplitude of the motion. Neither the work done, nor the amount of damping is dependent on the number of stops in the system.

\section{Concluding remarks}

We have described the behaviour of the system modelled throughout the parameter space. A new approach has been proposed to solve directly for the steady state behaviour of the system in regions of stick-slip motion. An explanation has been offered for the stop branch phenomena present in figure 3 . 


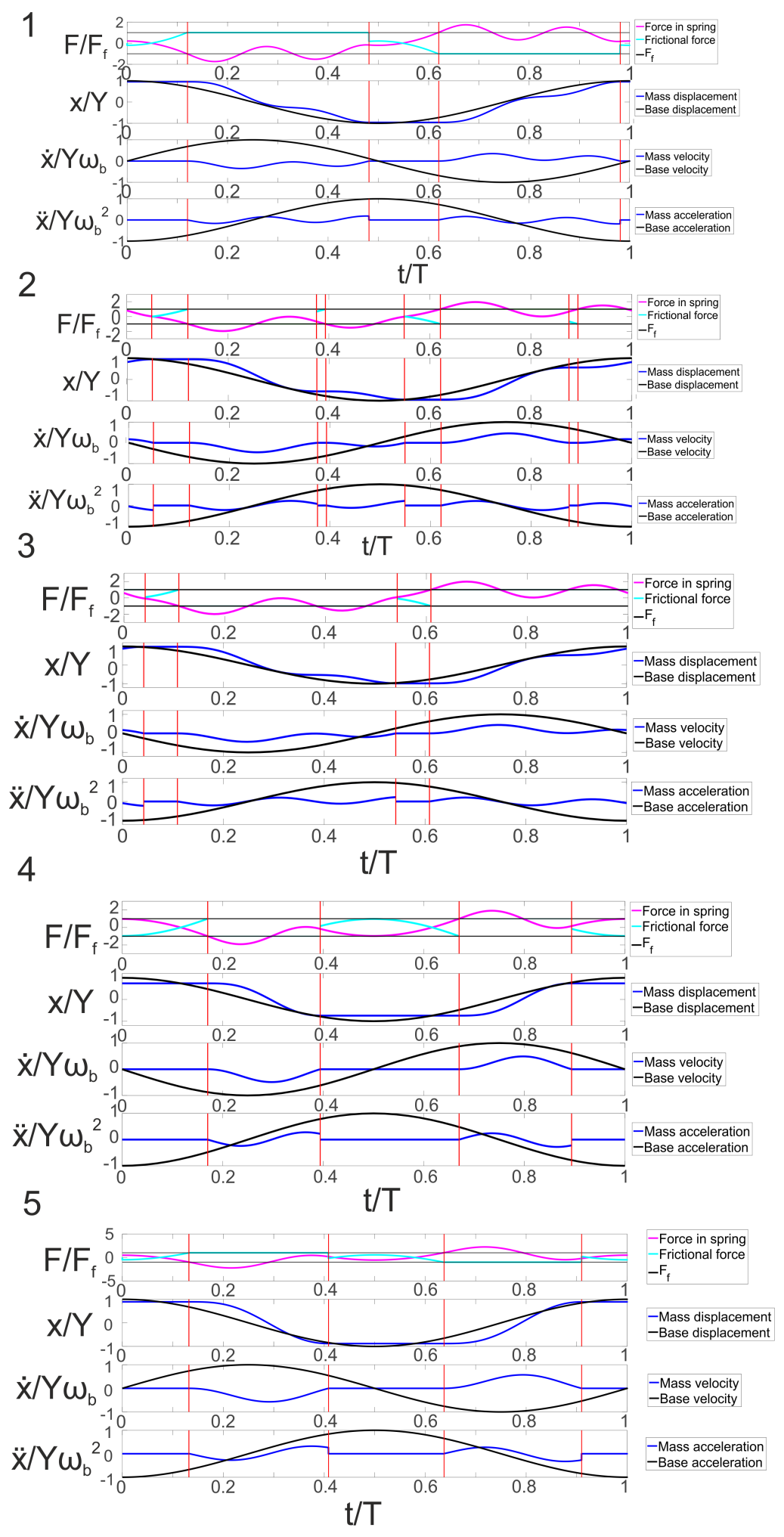

Figure 8: Five example cases showing the reasons for the branch. The coordiantes of each are $1:\left(\frac{k Y}{F_{f}}=4.5, r=0.2\right), 2:\left(\frac{k Y}{F_{f}}=4.5, r=0.25\right), 3:\left(\frac{k Y}{F_{f}}=4.8, r=0.25\right), 4:\left(\frac{k Y}{F_{f}}=3.75, r=0.25\right)$, $5:\left(\frac{k Y}{F_{f}}=4.5, r=0.3\right)$. 

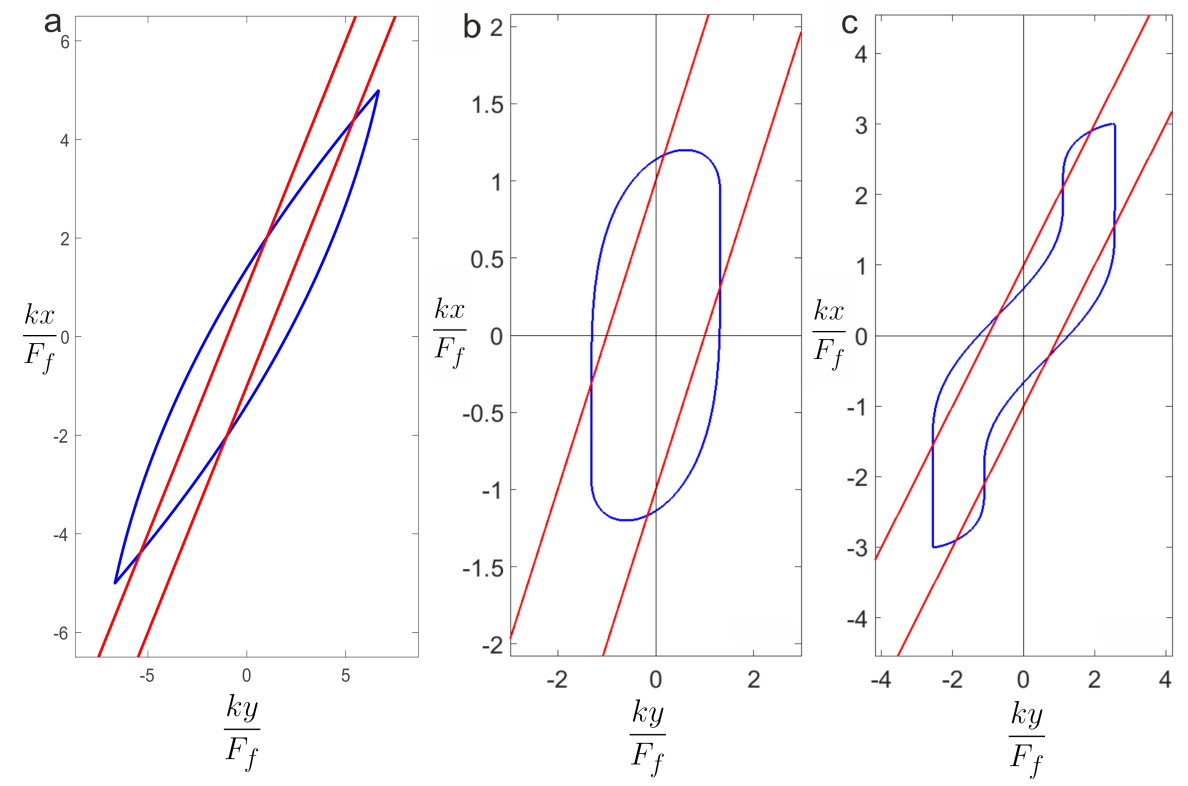

Figure 9: Dimensionless plots of normalised input displacement against response in cases of continuous and interrupted motion

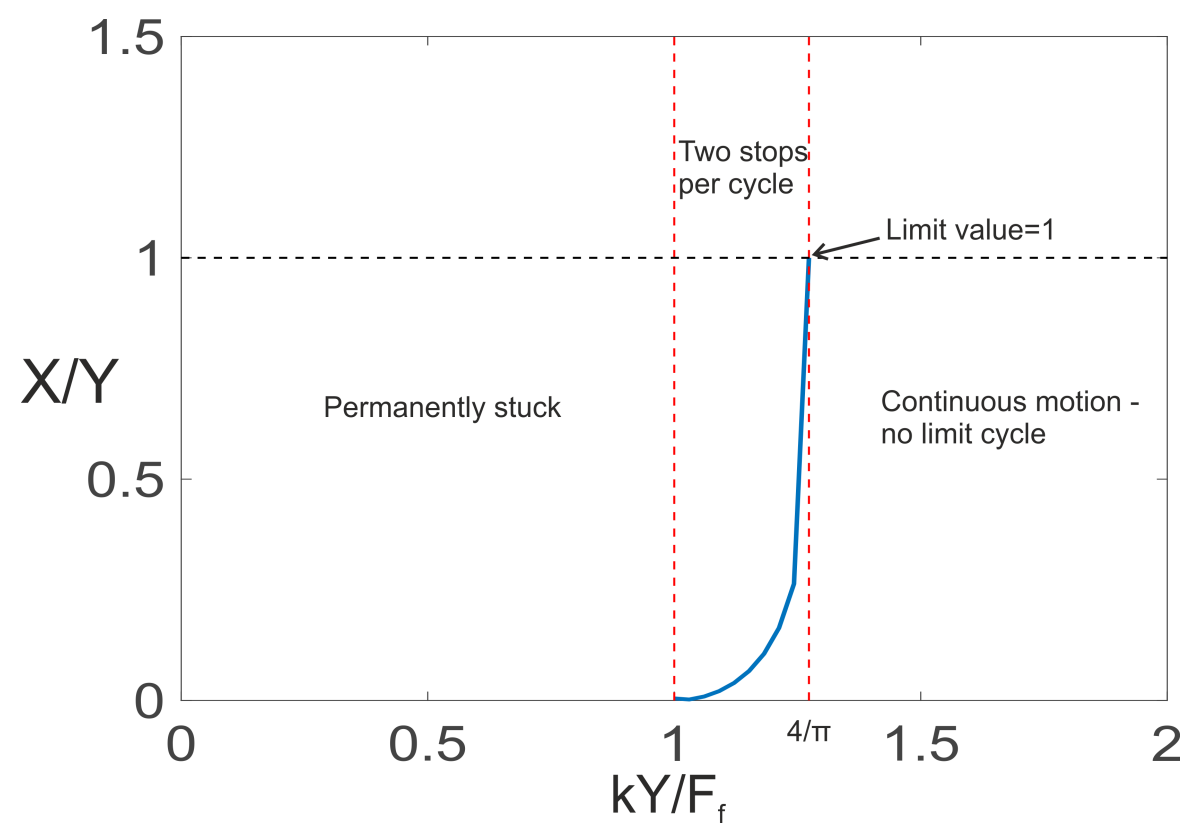

Figure 10: Plot of the resonant amplitude of the system 

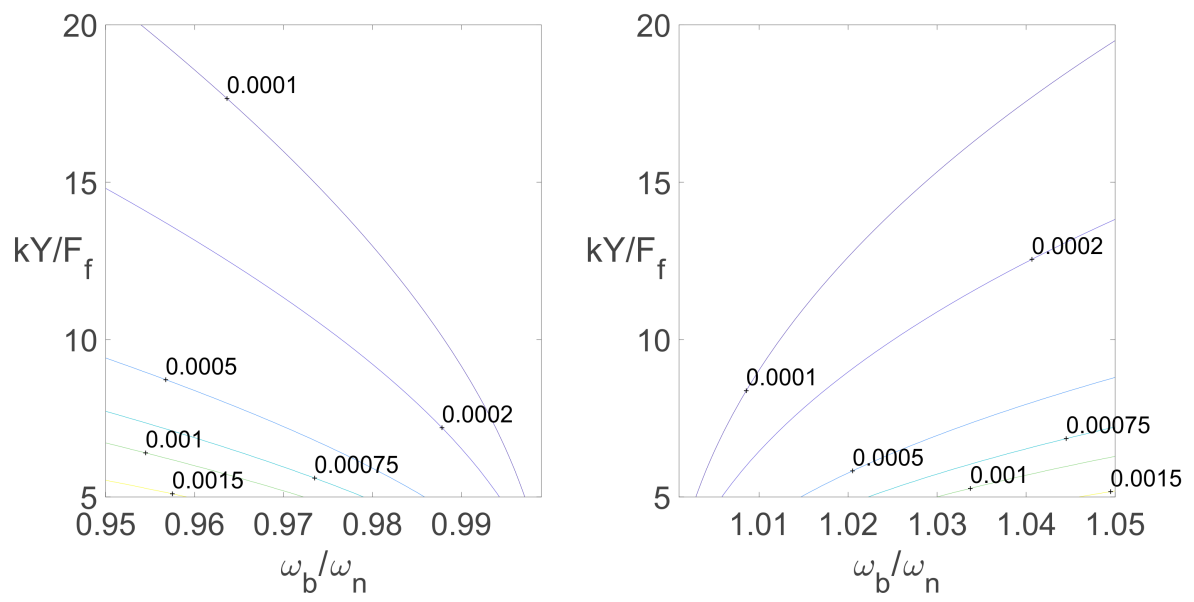

Figure 11: Error contours in the asymptotic solution

A new graphical procedure has been developed to easily identify features of the solution, including the number of stops, phase shift and relative velocity of the mass. The behaviour of the system at resonance has been explored and results of this investigation were presented, along with asymptotic solutions for the amplitude of the system near resonance. Further, comments were made about the energy balance and damping properties of the system and their dependence on features of the response.

So we are able to classify the stick/slip region of the solution by the number of periods of finite dwell per steady state cycle. In the regions of the parameter space studied, the transmissibilty of displacement is found using our method. The asymptotic solution found is a clear simplification of Den Hartog's solution near the system resonance.

Several issues remain unresolved, firstly, identification of the boundaries between stop numbers(ie. between two and four stops) is not tackled here. Closed form expressions for displacement transmissibility are also not found. One of the interesting findings of this work has been the identification of the stop branch, the uniqueness of this has not been further explored, and the boundaries around this branch present a further challenge to the identification of boundaries within the stick/slip region.

\section{Acknowledgements}

Daniel Riddoch is grateful for the financial support provided by Rolls-Royce for his internship. Alice Cicirello gratefully acknowledges the financial support provided by Balliol College for a Career Development Fellow- ship. David Hills and Alice Cicirello thanks Rolls-Royce plc and the EPSRC for the support under the Prosperity Partner- ship Grant "Cornerstone: Mechanical Engineering Science to Enable Aero Propulsion Futures", Grant Ref: EP/R004951/1. Part of the numerical investigations were performed in the Dynamics, Vibration and Uncertainty Lab (University of Oxford), which has been established thanks to the John Fell Fund (163/029).

The authors wish to thank an anonymous reviewer for highlighting the similarity of the approach of finding periodic solutions using boundary conditions, as discussed here, with the mathematical study of periodic orbits. This area of study is not in itself directly applicable to the modelled system, and the novelty of this work is in the determination of periodic solution for a problem involving a discontinuity. 


\section{References}

[1] Barber, J. R., Elasticity, Springer, 2010

[2] Barber, J. R., Contact Mechanics, Vol 250, Springer, 2018

[3] Johnson, K. L., Contact Mechanics, Cambridge University Press, 1985

[4] Hills, D. A., Mechanics of Fretting fatigue, Elsevier, 1994

[5] Den Hartog, J. P., Forced Vibrations with combined Viscous and Coulomb Damping, Philosophical Magazine, Volume 9(59), page 801-817 (1930)

[6] Hong H-K. and Liu C-S., Coulomb friction oscillator: Modelling and responses to Harmonic loads and base excitations, Journal of Sound and Vibration 229(5), page 1171-1192 (2000).

[7] Papangelo A. and Ciavarella M., On the limits of quasi-static analysis for a simple Coulomb frictional oscillator in response to harmonic loads, Journal of Sound and Vibration 339, page 280-289 (2014).

[8] Ma Y., Yu S. and Wang D., Vibration analysis of an oscillator with non-smooth dry friction constrain, Journal of Vibration and Control 23(14), page 2328-2344 (2015).

[9] Luo A. and Gegg B., Stick and non-stick periodic motions in periodically forced oscillators with dry friction, Journal of Sound and Vibration 291, page 132-168 (2006).

[10] Marino L., Cicirello A., and Hills D.A., Displacement transmissibility of a Coulomb friction oscillator subject to joined base-wall motion, Nonlinear Dyn (2019). https://doi.org/10.1007/s11071-019-04983-x 

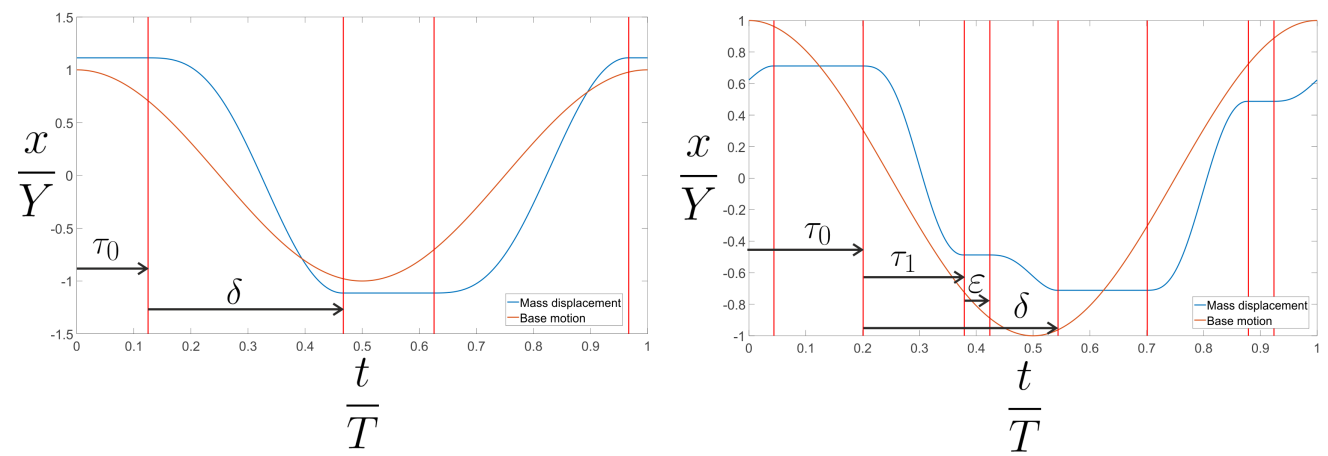

Figure 12: Definitions of time unknowns in different stop regimes

\section{A Boundary conditions}

The notation $x_{1}$ is used to denote motion with a negative velocity, $x_{2}$ has positive velocity. $x_{1,1}$ represents the first phase of motion with a negative velocity and so on. The unknowns used in section A.3 are defined in figure 12. In each case the response is referred to a base displacement cycle starting from its positive extreme.

Once the conditions are applied, the resulting simultaneous equations are solved in MATLAB, using the fsolve function and the Levenberg-Marquardt algorithm.

\section{A.1 No stops}

Here we lay out algebraically the conditions determined by Den Hartog, applied to both half-cycles of the steady state(denoted by $x_{1}$ and $x_{2}$ ) which allow us to solve directly for the steady state behaviour
1. $x_{1}(\phi)=x_{2}(\phi)$
5. $\dot{x}_{1}(\phi)=0$
2. $x_{1}\left(\phi+\frac{T}{2}\right)=x_{2}\left(\phi+\frac{T}{2}\right)$
6. $\dot{x}_{2}(\phi)=0$
3. $x_{1}(\phi)+x_{1}\left(\phi+\frac{T}{2}\right)=0$
7. $\dot{x}_{1}\left(\phi+\frac{T}{2}\right)=0$
4. $x_{2}(\phi)+x_{2}\left(\phi+\frac{T}{2}\right)=0$
8. $\dot{x}_{2}\left(\phi+\frac{T}{2}\right)=0$

\section{A.2 Two stops}

From the description of two stop systems, we are able to introduce boundary conditions constraining the two stop system. First we define two new unknowns. These allow us to replace $\phi$ from the original formulation as its meaning is no longer clear, as explained in section 3 . These two new unknowns $\tau_{0}$ and $\delta$ are shown in figure 12 and formally defined as follows:

$\tau_{0}$ - the time from the amplitude of the base displacement until the mass first slides, with negative velocity

$\delta$ - the time across each half-cycle that the mass is in motion.

With these new unknowns and the equations of motion, which are still valid for each period where the mass is sliding, symmetry and continuity are used to determine conditions across the cycle.

Consider first displacement. This has to be continuous and across a single cycle of the steady-state, is assumed to be symmetrical with respect to the half-period. In other words, the amplitude of response is equal in both the positive and negative directions. Moving to velocity, we know that at the start and end of each motion period, the velocity is zero. Finally the force in the spring must be precisely equal to the limiting value of the friction at the start of the motion period, and hence the acceleration at this point must be zero. These statements are set out algebraically now. 

1. $x_{1}\left(\tau_{0}\right)=x_{2}\left(\tau_{0}+\delta+\frac{T}{2}\right)$
7. $\dot{x}_{2}\left(\tau_{0}+\frac{T}{2}\right)=0$
2. $x_{1}\left(\tau_{0}+\delta\right)=x_{2}\left(\tau_{0}+\frac{T}{2}\right)$
8. $\dot{x}_{2}\left(\tau_{0}+\delta+\frac{T}{2}\right)=0$
3. $x_{1}\left(\tau_{0}\right)+x_{2}\left(\tau_{0}+\frac{T}{2}\right)=0$
9. $\ddot{x}_{1}\left(\tau_{0}\right)=0$
4. $x_{1}\left(\tau_{0}+\delta\right)+x_{2}\left(\tau_{0}+\delta+\frac{T}{2}\right)=0$
10. $\ddot{x}_{2}\left(\tau_{0}+\frac{T}{2}\right)=0$
5. $\dot{x}_{1}\left(\tau_{0}\right)=0$
11. $F_{\text {spring }}\left(\tau_{0}\right)=-\mu W$
6. $\dot{x}_{1}\left(\tau_{0}+\delta\right)=0$
12. $F_{\text {spring }}\left(\tau_{0}+\frac{T}{2}\right)=\mu W$

It should be noted, at this point, that these boundary conditions are insufficient to solve the problem analytically as Den Hartog did. A set of numerically stable solvers has been developed but these do require prior knowledge of the number of stops. This approach allows us to jump straight to the steady-state behaviour of the system.

\section{A.3 Four stops}

We can extend the two stop description to cover four stop cases. Again we must define more unknowns in time. We introduce $\tau_{1}$ and $\varepsilon$ :

- $\tau_{1}$ is the time taken for the mass to stick again having first started to slide;

- $\varepsilon$ is the duration of the intermediate stop.

Now we again apply continuity and symmetry as for the two-stop solution, and the following algebraic conditions result:
1. $x_{1,1}\left(\tau_{0}\right)=x_{2,2}\left(\tau_{0}+\delta+\frac{T}{2}\right)$
12. $\dot{x}_{2,1}\left(\tau_{0}+\frac{T}{2}\right)=0$
2. $x_{1,2}\left(\tau_{0}+\delta\right)=x_{2,1}\left(\tau_{0}+\frac{T}{2}\right)$
13. $\dot{x}_{2,1}\left(\tau_{0}+\tau_{1}+\frac{T}{2}\right)=0$
3. $x_{1,1}\left(\tau_{0}+\tau_{1}\right)=x_{1,2}\left(\tau_{0}+\tau_{1}+\varepsilon\right)$
4. $\begin{aligned} & x_{2,1}\left(\tau_{0}+\tau_{1}+\frac{T}{2}\right)= \\ & x_{2,2}\left(\tau_{0}+\tau_{1}+\varepsilon+\frac{T}{2}\right)\end{aligned}$
14. $\dot{x}_{2,2}\left(\tau_{0}+\tau_{1}+\varepsilon+\frac{T}{2}\right)=0$
5. $x_{1,1}\left(\tau_{0}\right)+x_{2,1}\left(\tau_{0}+\frac{T}{2}\right)=0$
15. $\dot{x}_{2,2}\left(\tau_{0}+\delta+\frac{T}{2}\right)=0$
6. $x_{1,2}\left(\tau_{0}+\delta\right)+$ $x_{2,2}\left(\tau_{0}+\delta+\frac{T}{2}\right)=0$
16. $\ddot{x}_{1,1}\left(\tau_{0}\right)=0$
7. $x_{1,1}\left(\tau_{0}+\tau_{1}\right)+$ $x_{2,1}\left(\tau_{0}+\tau_{1}+\frac{T}{2}\right)=0$
8. $\dot{x}_{1,1}\left(\tau_{0}\right)=0$
9. $\dot{x}_{1,1}\left(\tau_{0}+\tau_{1}\right)=0$
10. $\dot{x}_{1,2}\left(\tau_{0}+\tau_{1}+\varepsilon\right)=0$
11. $\dot{x}_{1,2}\left(\tau_{0}+\delta\right)=0$
17. $\ddot{x}_{1,2}\left(\tau_{0}+\tau_{1}+\varepsilon\right)=0$
18. $\ddot{x}_{2,1}\left(\tau_{0}+\frac{T}{2}\right)=0$
19. $\ddot{x}_{2,2}\left(\tau_{0}+\tau_{1}+\varepsilon+\frac{T}{2}\right)=0$
20. $F_{\text {spring }}\left(\tau_{0}\right)=-\mu W$
21. $F_{\text {spring }}\left(\tau_{0}+\tau_{1}+\varepsilon\right)=-\mu W$
22. $F_{\text {spring }}\left(\tau_{0}+\frac{T}{2}\right)=\mu W$
23. $F_{\text {spring }}\left(\tau_{0}+\tau_{1}+\varepsilon+\frac{T}{2}\right)=\mu W$ 